\title{
Suhuang Zhike Capsules for the Treatment of Cough Variant Asthma: A Meta-analysis
}

\author{
Cheng Gu $\left(\mathbb{D},{ }^{1}\right.$ Wenpan Peng $\left(\mathbb{D},{ }^{1}\right.$ Zhichao Wang $\mathbb{D}^{1},{ }^{1}$ Yong Xu $\left(\mathbb{D},{ }^{1}\right.$ Di Han $\mathbb{D},{ }^{1}$ \\ and Xianmei Zhou (iD ${ }^{1,2}$ \\ ${ }^{1}$ Affiliated Hospital of Nanjing University of Chinese Medicine, Nanjing 210029, China \\ ${ }^{2}$ Department of Respiratory Medicine, Jiangsu Province Hospital of Chinese Medicine, \\ Affiliated Hospital of Nanjing University of Chinese Medicine, Nanjing, Jiangsu 210029, China
}

Correspondence should be addressed to Xianmei Zhou; zhouxianmeijs@aliyun.com

Received 8 April 2020; Revised 18 December 2020; Accepted 21 December 2020; Published 31 December 2020

Academic Editor: Muhammad Nabeel Ghayur

Copyright (c) 2020 Cheng Gu et al. This is an open access article distributed under the Creative Commons Attribution License, which permits unrestricted use, distribution, and reproduction in any medium, provided the original work is properly cited.

Cough variant asthma (CVA) is a unique type of asthma characterized by cough as the only or primary clinical presentation. Inhaled glucocorticoid is the main treatment in clinical practice currently, but its efficacy remains relatively unsatisfactory. Traditional Chinese medicine has certain advantages in the treatment of CVA, and at present, the most commonly used traditional Chinese medicine is Suhuang Zhike Capsule (SZC). The aim of this study was to systematically evaluate the efficacy and safety of SZC in the treatment of CVA using a meta-analysis. A comprehensive search of papers published in the PubMed, Embase, Cochrane Library, China National Knowledge Infrastructure (CNKI), Chinese Biomedical Literature database (CBM), Wanfang Database, and VIP Information (VIP) from January 2018 to June 2019 was conducted. Review Manager 5.3 was used to carry out a meta-analysis of 10 studies that fulfilled the inclusion criteria. In a total of 10 randomized controlled trials, 896 CVA patients were included. The results showed the following: (1) compared with conventional Western medicine, SZC can effectively increase the efficacy rate of CVA (RR 1.25, 95\% CI, 1.16-1.35, $P<0.00001$ ) and (2) compared with other traditional Chinese medicines, SZC can effectively increase the efficacy rate of CVA (RR 1.44,95\% CI, 1.01-2.05, $P=0.05$ ), In conclusion, our study builds on existing clinical evidence showing that SZC is safe and effective in treating CVA. However, larger randomized controlled trials are required for further validation.

\section{Introduction}

Cough variant asthma (CVA) is a unique type of asthma that is characterized by cough as the only or primary clinical presentation. Most CVA patients do not show obvious signs of wheezing and shortness of breath and only have airway hyperresponsiveness. CVA mostly occurs at night or dawn and presents as a chronic, recurrent, and irritating dry cough that is exacerbated when hot/cold stimuli or smoke/foul smells are present [1]. Although CVA is not life threatening, its tendency to occur or exacerbate at night causes a lot of disruption to patients' lives. In addition, CVA tends to progress to classical asthma $[2,3]$. Both CVA and classical asthma share a similar pathogenesis involving the combined interaction between genetics, immunity, and environment and is mostly associated with airway inflammation [4], airway hyperresponsiveness [5-7], and airway remodeling $[8,9]$.

The principles for treating CVA are identical to classical asthma [10], and the recommended treatment guidelines are inhaled glucocorticoids combined with bronchodilators for, at least, 8 weeks [10], with some patients requiring longer term of treatment. Low oral doses of glucocorticoids are recommended for patients with severe airway inflammation or poor response to inhaled glucocorticoids. Leukotriene receptor antagonists can be used for a minority of patients that do not respond to inhaled glucocorticoids [11]. However, in consideration of the side effects of long-term usage of glucocorticoids, new treatment options are necessary.

Professor Enxiang Chao, one of the most famous specialists of traditional Chinese medicine, believed that the 
etiological mechanism of CVA is lung invasion by wind evil, lung qi obstruction, and airway contracture [12]. He developed SZC in order to clear the lungs and relieve coughing and to treat CVA in clinical practice in China. SZC is composed of Ephedra sinica Stapf, Beefsteak plant leaves and seeds, earthworms, loquat leaves, Periostracum cicadae, Peucedanum praeruptorum Dunn roots, Greater burdock seeds, and five-flavor berries. Modern pharmacological experiments showed that Beefsteak plant leaves, Ephedra sinica Stapf, and Periostracum cicadae in SZC have antiinflammatory, antitussive, and antiwheezing effects [13-16]. Currently, there are an increasing number of clinical trials using SZC to treat CVA, but most are small randomized controlled trials (RCTS) with small sample sizes, making it difficult to draw reliable conclusions. The aim of this metaanalysis is to assess the efficacy of SZC in the treatment of CVA from data collected in RCTs.

\section{Materials and Methods}

2.1. Publication Search Strategy. A search of papers published in the PubMed, Embase, Cochrane Library, China National Knowledge Infrastructure (CNKI), Chinese Biomedical Literature database (CBM), Wanfang Database, and VIP Information (VIP) from January 2018 to June 2019 was conducted. Two reviewers (Cheng Gu and Wenpan Peng) used the following keywords or free text terms to independently search papers in these electronic databases. The term used were "Suhuang Zhike capsules," "allergic cough," "variant cough," and "cough asthma" in Chinese and "cough variant asthma" and "cough-variance asthma" in Chinese and English. The authors of significant publications or experts in the relevant field were contacted for potential studies. Also, a search of unfinished research in the Cochrane central register of controlled trials, National Research Register (NRR), and clinical controlled trials (CTT) was conducted. A search of conference papers in ISTP, ISI proceedings, and OCLC Firstsearch proceedings was conducted. A search of the grey literature in GreyNet, Database of Abstracts of Reviews of Effects (DARE), and System for Information on Grey Literature in Europe (SIGLE) was conducted.

2.2. Inclusion Criteria. Studies to be included in a metaanalysis needed to meet all of the following criteria: (a) type of study: the type of the study needed to be RCTs which may have "RCT" in the title, abstract, or methods, its methods should have used a control group, randomization, allocation concealment, and all of the studies needed to be published from 1 January 2008 to 30 June 2019, (b) study subjects: patients with a definitive diagnosis of CVA of both genders and age $>18$ years, and (c) Intervention experiment: SZC only was used for the treatment group, and other drugs, placebo, or blank control were used for the control group.

2.3. Exclusion Criteria. The following studies were excluded: (a) SZC combined with other drugs were used for the treatment group in the original study, (b) studies in which the age of the study subjects was $<18$ years, and (c) republished papers, of which only one paper was used.

2.4. Markers. The primary prognostic markers were as follows: (a) Overall efficacy rate: clinical remission rate + marked improvement rate + improvement rate, (b) traditional Chinese medicine syndrome overall efficacy: clinical remission rate + marked improvement rate + improvement rate, (c) bronchial provocation test negativity conversion rate, and (d) adverse reactions.

The clinical efficacy for traditional Chinese medicine was classified as clinical remission, marked improvement, improvement, and not effective according to guidelines for clinical studies on new traditional Chinese medicine (Table 1) [17]. The nimodipine method was used to assess the efficacy for traditional Chinese medicine symptoms [18]. Efficacy was classified as clinical remission, marked improvement, improvement, and not effective (Table 2).

2.5. Study Selection and Data Extraction. The following information was collected from each study: (1) basic information, such as the first author's name and year of publication, (2) number of participants in each group and their gender, age, and medication duration, and (3) intervention measures and results of each experiment. The methodological quality of these studies was assessed by the two reviewers according to Cochrane Handbook for Systematic Reviews of Interventions 5.1.0, including (1) sequence generation, (2) allocation hiding, (3) blind method, (4) incomplete outcome data, (5) alternative outcome report, and (6) other sources of bias. Two researchers were responsible for independent screening and data extraction. Firstly, they read the title and abstract before reading the entire text of relevant papers. Next, cross validation was carried out by way of a third researcher who was responsible for judging and discussing the study when there were differences between the two researchers. The content of the data extracted included authors, year of publication, sample size, age and gender of subjects, detailed methodological information, detailed information on intervention measures, results, and adverse reactions.

2.6. Quality Evaluation. Two researchers assessed the methodological quality of the RCTs. The risk of the bias assessment tool recommended by the Cochrane manual was used to assess the risk of bias in the included papers. The content mainly included the randomization method, whether allocation was concealed, whether subjects and investigators were blinded, whether result assessors were blinded, whether results data were intact, and whether selective reporting was present. The actual situation of the included papers was then used to classify papers as low risk, high risk, and unclear.

2.7. Data Analysis. Review Manager 5.3 (Cochrane Collaboration, Oxford, United Kingdom) was used to combine data for meta-analysis. The combined RR with 95\% CI was 
TABLe 1: Traditional Chinese medicine clinical efficacy and its description.

\begin{tabular}{lc}
\hline Group & Description \\
\hline $\begin{array}{l}\text { Clinical remission } \\
\text { Marked }\end{array}$ & Complete remission of cough symptoms and scores of all primary symptoms were zero \\
Improvement & Coughing significantly alleviated or disappears. The scores of all primary symptoms decreased by 2 grades \\
Not effective & Coughing significantly alleviated. The scores of all primary symptoms decreased by 1 grade, or the score of 1 \\
symptom decreased by 2 grades while another symptom decreased by 1 grade \\
Coughing was not significantly alleviated or worsened.
\end{tabular}

TABle 2: Determination criteria for traditional Chinese medicine symptoms overall efficacy and its description.

\begin{tabular}{lc}
\hline Group & Description \\
\hline Clinical remission & $n \geq 95 \%$ \\
Marked improvement & $70 \% \leq n<95 \%$ \\
Improvement & $30 \% \leq n<70 \%$ \\
Not effective & $n<30 \%$ \\
\hline
\end{tabular}

Efficacy index $(n)=$ (pretreatment score-posttreatment score) $/$ pretreatment score $\times 100 \%$. 0 points: no cough, 3 points: occasional coughing, 6 points: frequent coughing, 9 points: persistent coughing.

calculated to compare binary and continuous variables. If heterogeneity was present in the combined studies, the random effects model (I $2>50 \%$ ) was used; otherwise, the fixed effects model was used. A difference of $P<0.05$ was considered to be statistically significant. If there were more than 10 studies included in the meta-analysis, Stata 14.0 was used for the Egger test and Begg test to detect publication bias.

\section{Results}

3.1. Search Results. One hundred and four potentially related papers were selected based on the predefined search criteria, of which 22, 30, 25, and 27 papers were from the CNKI, VIP, Wanfang database, and CBM, respectively. Following that, EndNote X8 was used to exclude 80 repeated papers. After reading the title and abstract, 9 papers involving children were excluded. Following that, 2 reviewers read the full text of the remaining 15 papers, and 5 papers involved combinational drugs and were, therefore, excluded. Therefore, a total of 10 qualified trials were used for the current metaanalysis. A total of 896 patients participated in these 10 studies, of which 535 were from the SZC group and 361 were from the control group (Figure 1 and Table 3).

3.2. Methodological Evaluation of the Included Studies. All included studies mentioned randomization, but only 2 studies $[19,20]$ described the specific randomization method, and 2 papers $[19,20]$ mentioned allocation concealment and blinding. The remaining studies [21-28] did not mention randomization and blinding, and we were unable to determine whether selective reporting bias was present. A total of 3 trials $[19,20,23]$ mentioned the shedding and removal of cases, none of which were lost to follow-up, while the rest $[21,22,24-28]$ were not mentioned, but their results were complete and other biases were unclear. Figure 2 lists the detailed information on the methodological quality of all included studies.

\subsection{Meta-Analysis of SZC in the Treatment of Cough Variant Asthma}

3.3.1. Efficacy Rate. (1) Comparison of SZC with conventional Western medicine: as shown in Figure 3, 8 studies examined the differences in the efficacy rate between SZC and Western medicine $[19,21-25,27,28]$. There were 571 patients included in total, including 262 in the control group and 309 in the treatment group. As there was no heterogeneity in the 8 studies (chi-square $=3.73, P=0.81, \mathrm{I}^{2}=0 \%$ ), the fixed effects model was used for statistical analysis. The results of the study showed that compared with conventional Western medicine, SZC can effectively increase the efficacy rate (RR 1.25, 95\% CI: 1.16-1.35, $P<0.00001$ ) (Figure 3).

(2) Comparison of SZC with other traditional Chinese medicines: as shown in Figure 4, 2 studies examined the differences in the efficacy rate between SZC and Western medicine $[20,26]$. There were 325 patients included in total, including 99 in the control group and 226 in the treatment group. As there was heterogeneity in the 2 studies (chisquare $=3.22, P=0.07, \mathrm{I}^{2}=69 \%$ ), the random effects model was used for statistical analysis. The results of the study showed that compared with other traditional Chinese medicine, SZC can effectively increase efficacy (RR 1.44, 95\% CI: 1.01-2.05, $P=0.05$ ) (Figure 4).

3.3.2. Traditional Chinese Medicine Symptom Efficacy Rate. Three studies examined the traditional Chinese medicine symptom efficacy rate $[19,20,23]$. There were 351 patients included in total, including 104 in the control group and 247 in the treatment group. Since heterogeneity was shown in three studies (chi-square $=66.89, P<0.00001, \mathrm{I}^{2}=97 \%$ ), the random effects model was used for statistical analysis. Results showed that there was no significant difference between the SZC and control groups (RR 1.43, 95\% CI:0.58-3.53. $P<0.00001$ ) (Figure 5).

\subsubsection{Bronchial Provocation Test Negativity Conversion Rate.} Three studies examined the bronchial provocation test negativity conversion rate $[19,20,23]$. There were 242 patients included in total, including 70 in the control group and 172 in the treatment group. Since heterogeneity was shown in 3 studies (chi-square $=4.30, P=0.12, \mathrm{I}^{2}=54 \%$ ), the random effects model was used for statistical analysis. Results showed that there was no significant difference between the SZC and control groups (RR 1.37, 95\% CI: 0.52-3.64. $P=0.52$ ) (Figure 6). 


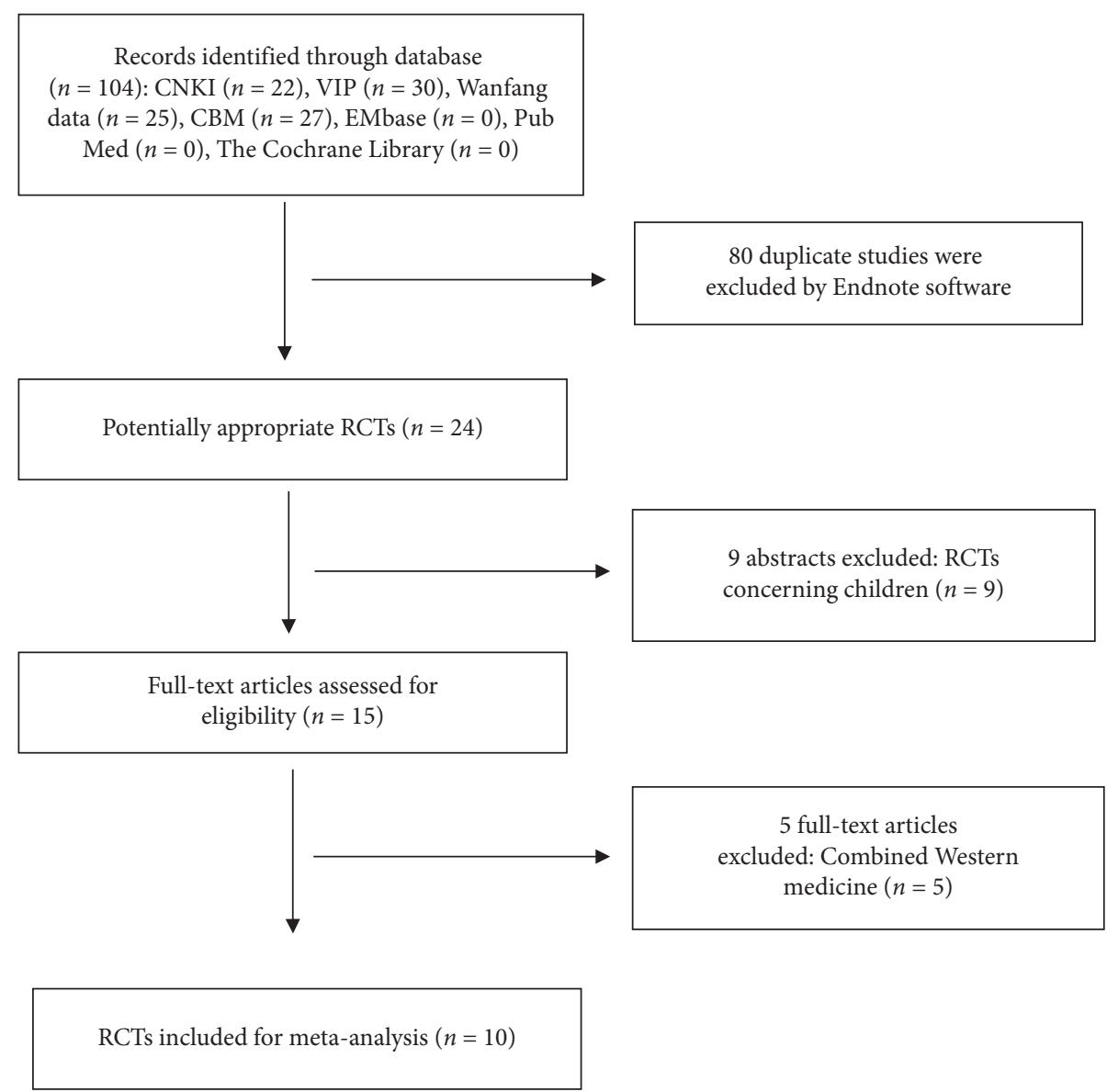

FIgURE 1: Flow diagram of the literature search process.

TABle 3: Characteristics of studies conforming to criteria.

\begin{tabular}{|c|c|c|c|c|c|c|}
\hline \multirow{2}{*}{ Included studies } & \multicolumn{2}{|c|}{$\mathrm{N}$} & \multicolumn{2}{|r|}{ Intervention measures } & \multirow{2}{*}{ Treatment course } & \multirow{2}{*}{ Results } \\
\hline & $\mathrm{T}$ & $\mathrm{C}$ & $\mathrm{T}$ & $\mathrm{C}$ & & \\
\hline Zhang, 2008 & 196 & 69 & SZC & Zhike Ningsou capsules & $14 \mathrm{~d}$ & (1) (2) (4) (6) (7) \\
\hline Rong, 2012 & 30 & 30 & SZC & Compound liquorice tablets & $14 \mathrm{~d}$ & (1) \\
\hline Jia, 2012 & 38 & 16 & SZC & Fluticasone/salmeterol inhalation powder & $28 \mathrm{~d}$ & (1) (2) (3) (4) (5) (6) (7) \\
\hline Huang, 2013 & 32 & 32 & SZC & Montelukast sodium & $14 \mathrm{~d}$ & (1) \\
\hline Liu, 2013 & 32 & 28 & SZC & Ketotifen & $7-14 d$ & (1) (7) \\
\hline Guo, 2014 & 40 & 40 & SZC & Salbutamol nebulization solution & $14 \mathrm{~d}$ & (1) (7) \\
\hline Ge, 2015 & 40 & 20 & SZC & Fluticasone/salmeterol inhalation powder & $28 \mathrm{~d}$ & (1) (2) (3) (4) (5) (6) (7) \\
\hline Yao, 2016 & 40 & 40 & SZC & Montelukast sodium & $14 \mathrm{~d}$ & (1) \\
\hline $\mathrm{Lu}, 2016$ & 38 & 38 & SZC & Montelukast sodium & $14 \mathrm{~d}$ & (1) (2) (4) \\
\hline Sun, 2017 & 49 & 49 & SZC & Montelukast sodium & $14 \mathrm{~d}$ & (1) (7) \\
\hline
\end{tabular}

Results: (1) overall efficacy for cough; (2)traditional Chinese medicine overall efficacy; (3)traditional Chinese medicine syndrome overall efficacy; (4)eosinophil count; (5)serum IgE level; (6) bronchial provocation test negativity conversion rate; (7)safety analysis.

3.3.4. Adverse Reactions. Only 1 patient developed nausea and vomiting after taking SZC, and no other adverse reactions were reported [28]. The common adverse reactions of Western medicine or traditional Chinese medicine in the control group were headache, dizziness, drowsiness, fatigue, dry mouth, muscle tremor, accelerated heart rate, and oral ulcer. However, some of the literature do not specify the number of adverse events reported, it is difficult to calculate the total incidence of adverse reactions.

3.3.5. Publication Bias Analysis. As shown in Figure 7, funnel plot analysis was carried out using the efficacy rate. The funnel plot shows that poor symmetry and 


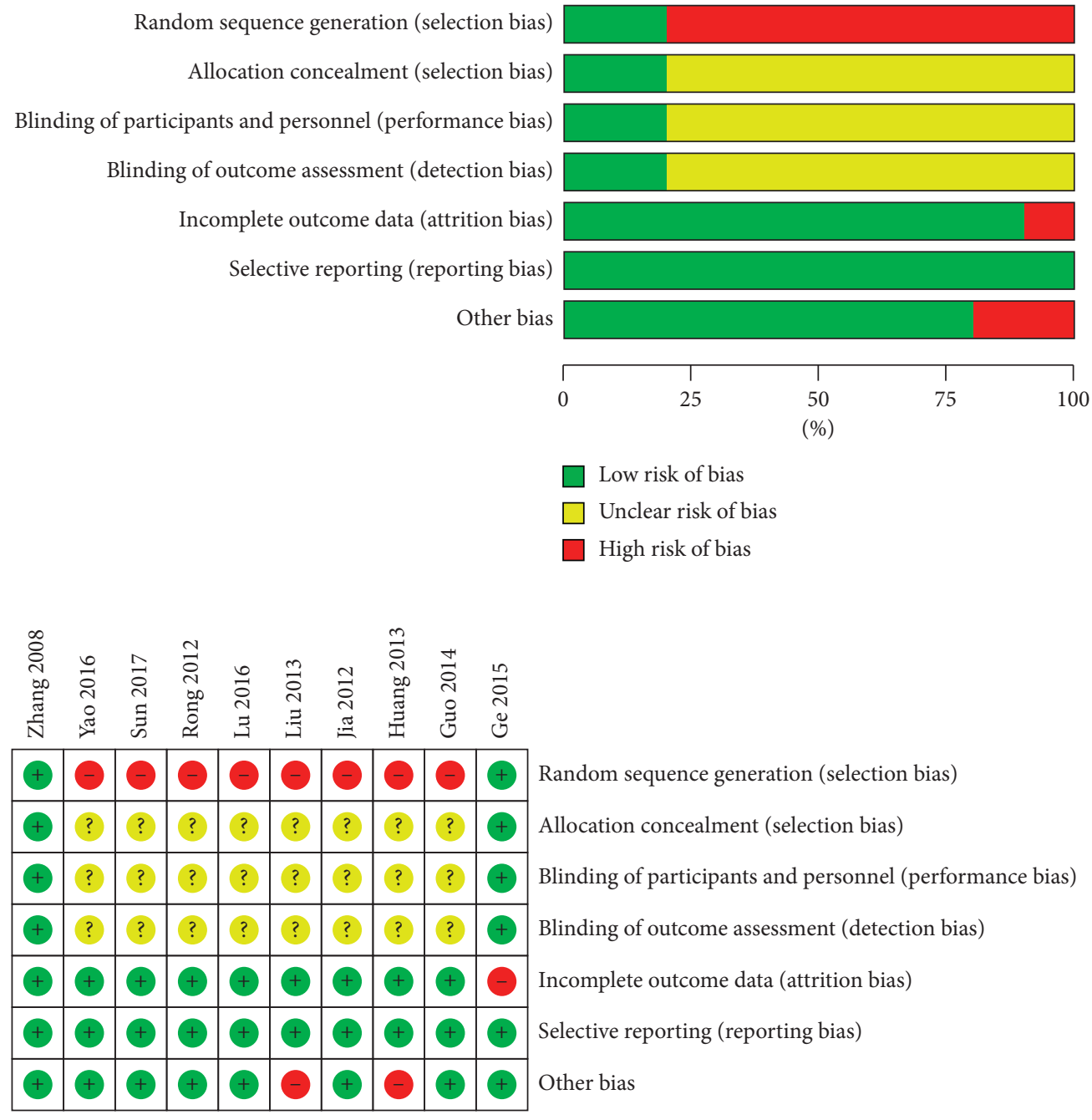

FIgURE 2: Risk of bias graph and risk of bias summary.

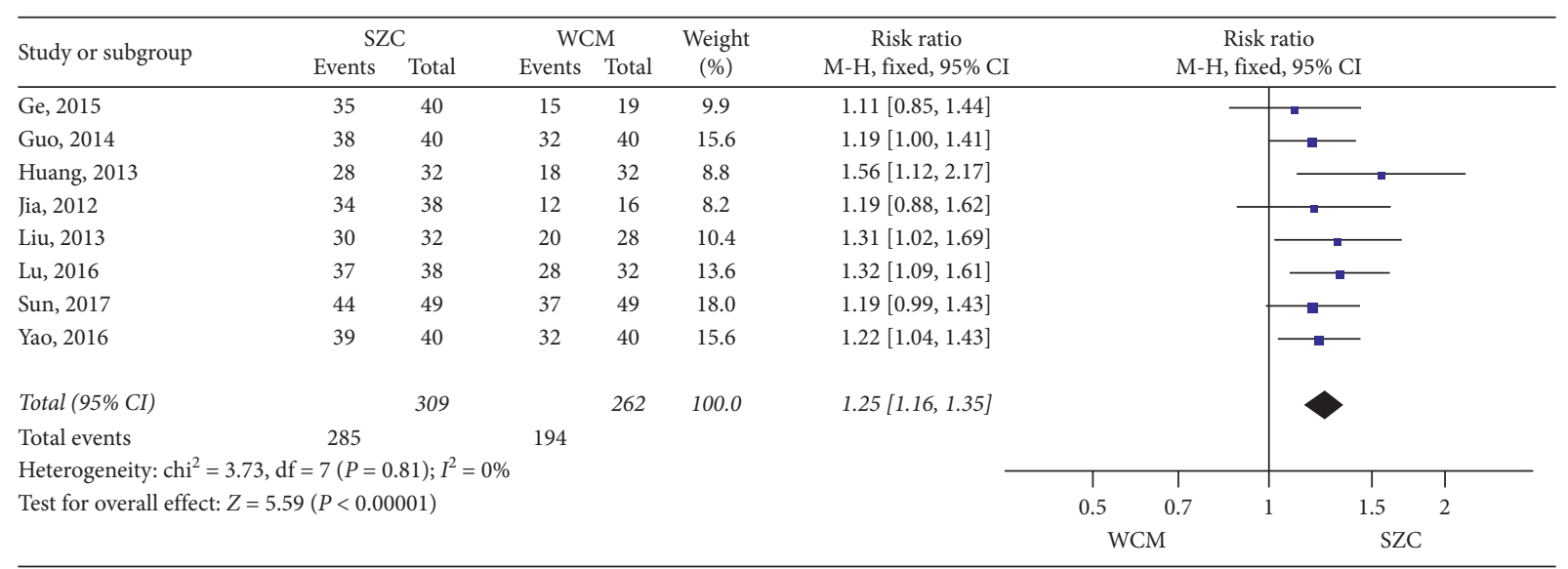

FIGURE 3: The efficacy rate using SZC versus conventional Western medicine.

publication bias may be present. Stata 14.0 was used for Egger and Begg tests on the 10 studies, which showed that publication bias was absent $(P=0.072, P=0.210$, respectively) (Figure 7).

\section{Discussion}

The present study describes a meta-analysis of RCTs investigating the efficacy of SZC to treat CVA. A total of 10 


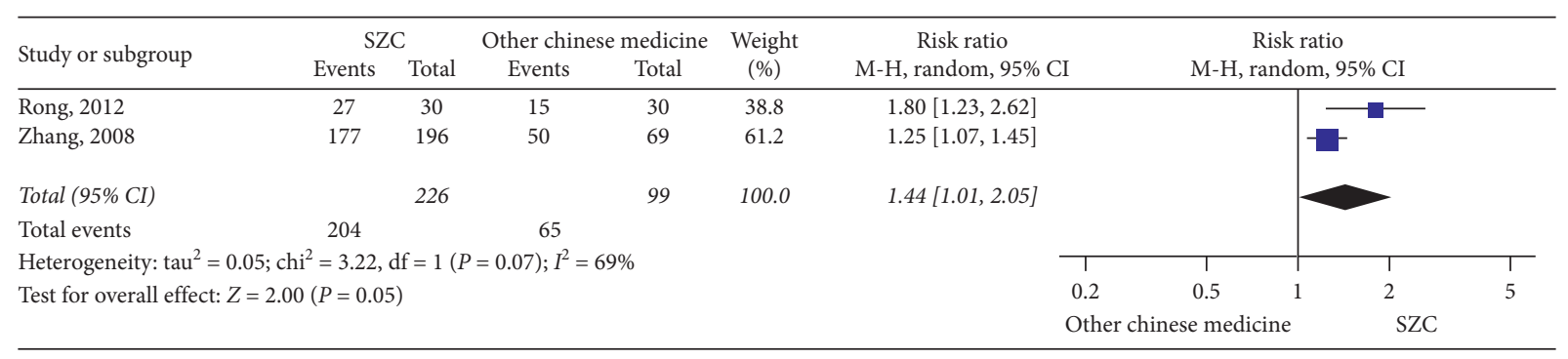

FIGURE 4: The efficacy rate using SZC versus other traditional Chinese medicine.

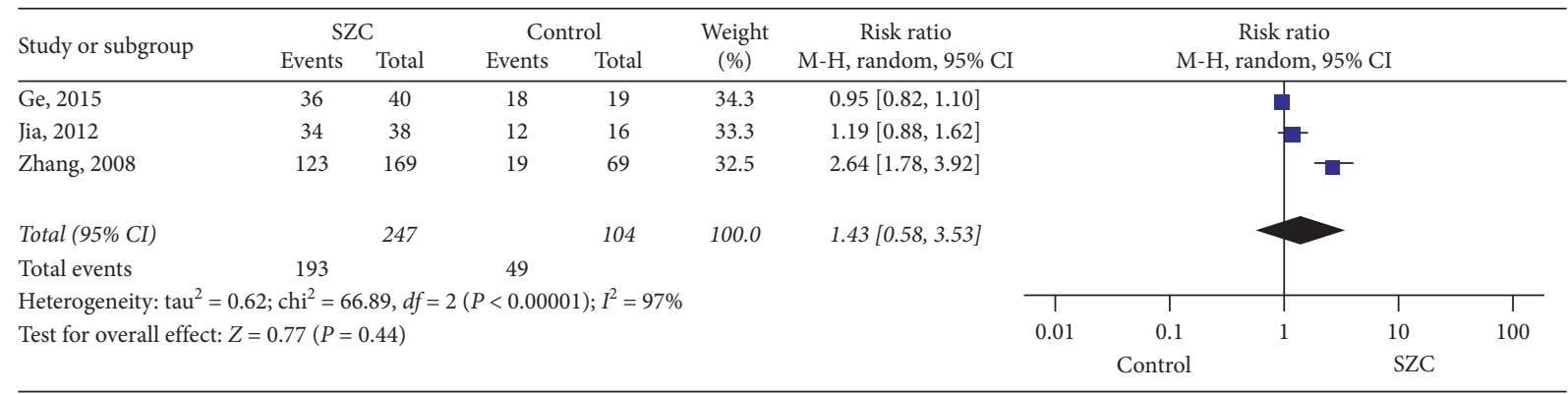

Figure 5: Traditional Chinese medicine symptom efficacy rate.

\begin{tabular}{|c|c|c|c|c|c|c|c|c|c|}
\hline \multirow{3}{*}{$\begin{array}{l}\text { Study or subgroup } \\
\text { Ge, } 2015\end{array}$} & \multicolumn{2}{|c|}{ SZC } & \multicolumn{2}{|c|}{ Control } & \multirow{2}{*}{$\begin{array}{c}\text { Weight } \\
(\%)\end{array}$} & \multirow{2}{*}{$\begin{array}{c}\text { Risk ratio } \\
\text { M-H, random, 95\% CI }\end{array}$} & \multirow{2}{*}{\multicolumn{2}{|c|}{$\begin{array}{c}\text { Risk ratio } \\
\mathrm{M}-\mathrm{H} \text {, random, } 95 \% \mathrm{CI}\end{array}$}} & \\
\hline & \multirow{2}{*}{$\frac{\text { Events }}{10}$} & \multirow{2}{*}{$\frac{\text { Total }}{40}$} & \multirow{2}{*}{$\frac{\text { Events }}{6}$} & \multirow{2}{*}{$\begin{array}{c}\text { Total } \\
19\end{array}$} & & & & & \\
\hline & & & & & 43.1 & $0.79[0.34,1.86]$ & $\square$ & \multirow{2}{*}{+} & \\
\hline Jia, 2012 & 12 & 38 & 4 & 16 & 39.3 & $1.26[0.48,3.33]$ & & & \\
\hline Zhang, 2008 & 17 & 94 & 1 & 35 & 17.6 & $6.33[0.87,45.80]$ & & & \\
\hline \multicolumn{2}{|l|}{ Total (95\% CI) } & 172 & & 70 & 100.0 & $1.37[0.52,3.64]$ & & & \\
\hline Total events & 39 & & 11 & & & & & & \\
\hline \multicolumn{6}{|c|}{ Heterogeneity: $\operatorname{tau}^{2}=0.39 ; \mathrm{chi}^{2}=4.30, d f=2(P=0.12) ; I^{2}=54 \%$} & $\Gamma$ & 1 & T & \\
\hline \multicolumn{6}{|c|}{ Test for overall effect: $Z=0.64(P=0.52)$} & 0.01 & 0.1 & 10 & 100 \\
\hline & & & & & & & Contro & SZC & \\
\hline
\end{tabular}

FIGURE 6: Bronchial provocation test negativity conversion rate.

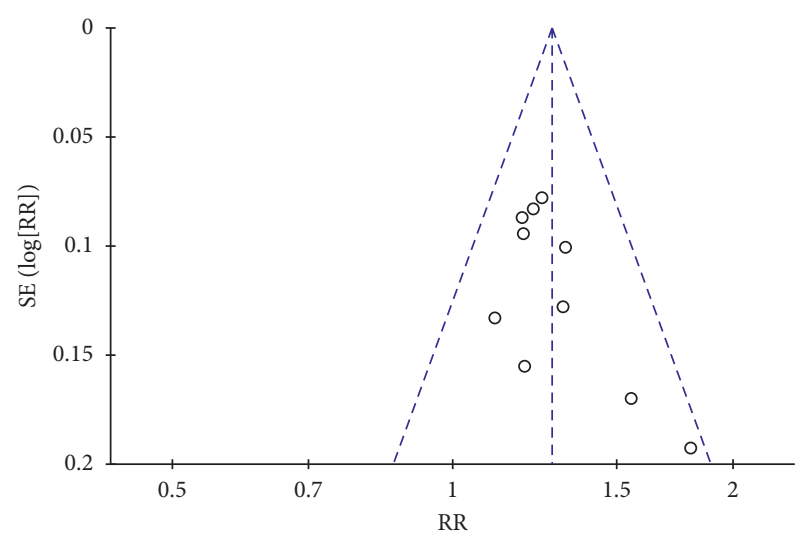

FIGURE 7: Funnel plots for assessing publication bias.

randomized controlled trials were included in this study, including 896 CVA patients. Results showed that the efficacy rate of SZC in the treatment of CVA is superior to conventional Western medicines and other traditional Chinese medicines, and the incidence of adverse reactions is lower than that in the control groups.

Our study confirms the results of Zhang et al. [29], which showed that SZC combined with Western medicine had a significant effect on CVA, suggesting that SZC might have a positive effect on CVA treatment. In terms of adverse reactions, SZC had fewer adverse reactions than salmeteroticasone combined with montelukast [30], suggesting its relative safety. However, there are some limitations of this study, suggesting that further research is required. First, the sample sizes of the RCTs included were very small, and it is difficult to rule out the influence of contingency factors. The clinical trial protocols of most of the included studies were still not stringent enough; most used symptom markers, and few studies used eosinophil counts, which means it is not objective enough. The reasons for heterogeneity may include the following three points: first, the drugs in the control group were inconsistent, some were inhaled and some were oral Chinese medicine; second, the treatment course was different, some were 14 days and some were 28 days; and third, the methodology might be different, such as some used 
blinding and some did not use blinding. In addition, the overall methodological quality of the RCTS included was not high; for example, only 3 studies described randomization and allocation concealment, while only 3 studies described blinding. In addition, follow-up was not described in the included studies, and there was no long-term efficacy evaluation of SZC in the treatment of CVA.

\section{Conclusions}

The current evidence shows that SZC is effective in treating CVA. However, due to the limitations of this study, we recommend that future traditional Chinese medicine clinical trials should be conducted as standardized, multicenter, and large sample size high-quality randomized, double-blind trials to more accurately assess its efficacy and safety.

\section{Data Availability}

The extracted data used to support the findings of this study are available from the corresponding author upon request.

\section{Conflicts of Interest}

All authors declared that there are no conflicts of interest regarding the publication of this paper.

\section{Acknowledgments}

The authors would like to thank Editage (http://www. editage.cn) for English language editing. This study was sponsored by the National Natural Science Foundation (No. 81673936) and Jiangsu Provincial Graduate Student Research and Practice Innovation Project Item (KYCX19_1192).

\section{References}

[1] G. Jiang, X. Huang, T. Li, and D. Xu, "Chronic cough: clinical characteristics and etiologies of 510 cases," Turkish Journal of Medical Sciences, vol. 46, no. 6, pp. 1734-1739, 2016.

[2] M. Fujimura, H. Ogawa, Y. Nishizawa et al., "Comparison of atopic cough with cough variant asthma: is atopic cough a precursor of asthma?” Thorax, vol. 58, no. 1, pp. 14-18, 2003.

[3] Q. Miao, P.-c. Wei, M.-r. Fan, and Y.-p. Zhang, "Clinical study on treatment of cough variant asthma by Chinese medicine," Chinese Journal of Integrative Medicine, vol. 19, no. 7, pp. 539-545, 2013.

[4] G. Terasaki and D. S. Paauw, "Evaluation and treatment of chronic cough," Medical Clinics of North America, vol. 98, no. 3, pp. 391-403, 2014.

[5] H. Matsumoto, A. Niimi, M. Takemura et al., "Features of cough variant asthma and classic asthma during methacholine-induced brochoconstriction: a cross-sectional study," Cough, vol. 5, no. 3, 2009.

[6] A. Niimi, R. Amitani, K. Suzuki, E. Tanaka, T. Murayama, and F. Kuze, "Eosinophilic inflammation in cough variant asthma," European Respiratory Journal, vol. 11, no. 5, pp. 1064-1069, 1998.

[7] Y. Obase, T. Shimoda, R. Kishikawa et al., "Trigger of bronchial hyperresponsiveness development may not always need eosinophilic airway inflammation in very early stage of asthma," Allergy \& Rhinology (Providence), vol. 7, no. 1, pp. 1-7, 2016.

[8] H. Matsumoto, A. Niimi, R. P. Tabuena et al., "Airway wall thickening in patients with cough variant asthma and nonasthmatic chronic cough," Chest, vol. 131, no. 4, pp. 1042-1049, 2007.

[9] A. Niimi, H. Matsumoto, M. Minakuchi, M. Kitaichi, and R. Amitani, "Airway remodelling in cough-variant asthma," The Lancet, vol. 356, no. 9229, pp. 564-565, 2000.

[10] Chinese Thoracic Society Asthma Consortium, "Diagnosis and treatment guidelines for cough (2015)," Chinese Journal of Tuberculosis and Respiratory Diseases, vol. 39, no. 5, pp. 323-354, 2016.

[11] Chinese Thoracic Society Asthma Consortium, "Diagnosis and treatment guidelines for bronchial asthma (2016)," Chinese Journal of Tuberculosis and Respiratory Diseases, vol. 39, no. 9, pp. 675-697, 2016.

[12] J. Q. Li, Y. Han, Z. D. Zhang et al., "Chao En-Xiang's experience of treating cough caused by wind pathogen," Jiangxi Journal of Traditional Chinese Medicine, vol. 41, no. 7, pp. 13-14, 2010.

[13] X. Chu, Z. Xu, D. Wu et al., "In vitro and in vivo evaluation of the anti-asthmatic activities of fractions from pheretima," Journal of Ethnopharmacology, vol. 111, no. 3, pp. 490-495, 2007.

[14] K. Minamizawa, H. Goto, Y. Shimada, K. Terasawa, and A. Haji, "Effects of eppikahangeto, a Kampo formula, and Ephedrae herba against citric acid-induced laryngeal cough in Guinea pigs," Journal of Pharmacological Sciences, vol. 101, no. 2, pp. 118-125, 2006.

[15] L. Yang, G.-Y. Li, Q.-R. Li, and J.-H. Wang, “Two new $\mathrm{N}$-acetyldopamine tetrapolymers from Periostracum cicadae," Journal of Asian Natural Products Research, vol. 14, no. 3, pp. 204-209, 2012.

[16] J. Z. Wang, S. C. Tao, Y. C. Xi et al., "Pharmacological effects of Zisu and Baisu," China Journal of Chinese Material Medica, vol. 22, no. 1, pp. 49-64, 1997.

[17] X. Y. Zheng, Guidelines for Clinical Trials on New Traditional Chinese Medicines (Interim), China Medical Science Press, Beijing, China, 2002.

[18] People's Republic of China Ministry of Health, Guidelines for Clinical Trials on New Traditional Chinese Medicines, People's Republic of China Ministry of Health, Beijing, China, First Edition, 1993.

[19] Y. T. Ge, Clinical Observations of Suhuang Zhike Capsules in the Treatment of Cough Variant Asthma, Beijing University of Chinese Medicine, Beijing, China, 2015.

[20] Y. P. Zhang, Q. Miao, Y. Chao et al., "A multi-centered, randomized-controlled clinical study on Suhuang Zhike capsule for cough variant asthma," Journal of Traditional Chinese Medicine, vol. 6, pp. 504-506, 2008.

[21] L. S. Guo, " 80 cough variant asthma cases treated by Suhuang Zhike capsules," Chinese Medicine Modern Distance Education of China, vol. 12, no. 1, pp. 42-43, 2014.

[22] W. M. Huang and H. M. Ma, "Efficacy observations of 64 cough variant asthma cases treated by Suhuang Zhike capsules," Guide of China Medicine, vol. 11, no. 15, pp. 290-291, 2013.

[23] M. Y. Jia, Clinical Efficacy Assessment of Suhuang Zhike Capsules in the Treatment of Cough Variant Asthma, Beijing University of Chinese Medicine, Beijing, China, 2012.

[24] Y. Liu, "Clinical study on Suhuang Zhike capsule in treatment of cough variant asthma," China Modern Doctor, vol. 51, no. 10, pp. 137-138, 2013. 
[25] C. Y. Lu, S. Z. Wei, S. N. Chen et al., "Thirty-eight cases of cough variant asthma treated with suhuang cough capsule," Henan Traditional Chinese Medicine, vol. 36, no. 8, pp. 1487-1488, 2016.

[26] Y. X. Rong, M. H. Xie, and L. Jin, "Efficacy observations of Suhuang Zhike capsules in the treatment of 30 cough variant asthma patients," Guide of China Medicine, vol. 10, no. 21, p. 624, 2012.

[27] Y. H. Sun, Y. X. Wang, and X. M. Ma, "Analysis of Su Huang cough capsule in treating cough variant asthma," Studies of Trace Elements and Health, vol. 34, no. 6, pp. 7-9, 2017.

[28] Y. Yao, "Efficacy analysis of Suhuang Zhike capsules in the treatment of cough variant asthma," The Medical Forum, vol. 20, no. 29, pp. 4087-4088, 2016.

[29] T. Zhang, Y. H. Li, G. X. Liu et al., "Systematic evaluation and meta-analysis of Suhuang Zhike capsule in the treatment of cough-variant asthma," Yunnan Journal of Traditional Chinese Medicine, vol. 6, pp. 89-92, 2019.

[30] W. T. Feng, H. Yi, and H. Y. Yuan, "Efficacy and safety of salmeterol/fluticasone combined with montelukast versus salmeterol/fluticasone inthe treatment of cough variant asthma: A meta-analysis," China Pharmacy, vol. 5, pp. 699-703, 2018. 